\title{
A rotating disk apparatus for assessing the biodegradation of polycyclic aromatic hydrocarbons transferring from a non-aqueous phase liquid to solutions of surfactant Brij 35
}

\author{
Letícia A. Bernardez
}

Received: 9 June 2008/Accepted: 27 August 2008/Published online: 17 September 2008

(C) Springer-Verlag 2008

\begin{abstract}
A rotating disk apparatus was used to investigate the biodegradation of PAHs from non-aqueous phase liquids to solutions of Brij 35. The mass transfer of PAHs in absence of surfactant solution was not large enough to replenish the degraded PAHs. The addition of surfactant resulted in an overall enhancement of biodegradation rates compared to that observed in pure aqueous solution. This is because surfactant partition significant amount of PAHs into the bulk phase, where uptake occurs but the supply of PAHs to the aqueous phase through micellar solubilization at latter period limited biodegradation rates. It was demonstrated the relationship between biodegradation rate and surfactant dose and the mechanisms controlling the mass transfer of PAH from NAPLs. The satisfactory comparison of the experimental data with the predictions of a model, which parameters were determined from independent solubilization and dissolution experiments and based on the main assumption that the solutes must be present in the true aqueous phase to be degraded, allows us to conclude the absence of direct uptake of PAHs by bacteria.
\end{abstract}

Keywords Dissolution - Biodegradation · PAHs · Surfactant $\cdot$ Rotating disk

\section{A. Bernardez}

Department of Civil Engineering and Applied Mechanics, McGill University, Montreal, QC H3A 2K6, Canada

Present Address:

L. A. Bernardez ( $\square)$

Departamento de Ciências da Biointeração, ICS,

Universidade Federal da Bahia, C.P. 6974, Salvador,

Bahia 41810-971, Brazil

e-mail: Leticia.Bernardez@mail.mcgill.ca

\section{Introduction}

Many sites have been contaminated by chemically complex non-aqueous phase liquids (NAPLs) such as coal tar, creosote, and petroleum liquids from accidental spills and leaks that contain significant fraction of polycyclic aromatic hydrocarbons (PAHs) [1]. Polycyclic aromatic hydrocarbons (PAHs) are formed by the incomplete combustion or thermal decomposition (pyrolysis) of organic material and are widely distributed in the environment. A group of PAHs and their derivatives are toxicologically significant. Their mutagenic or carcinogenic properties are the main risk for human health. PAHs are generally slightly water-soluble and have low volatility and thus are difficult to extract or eliminate at NAPL-contaminated sites.

Most PAHs are biodegradable [2]. Bacterial strains living in contaminated soils are in fact able to degrade organic xenobiotic substances thanks to specific catabolic pathways. Adaptive mechanisms allow bacteria to use xenobiotics as their main carbon source. Contradictory results are reported in the literature on the efficiency of bacterial conversion in the presence of surfactants. For instance, some authors report an enhancement of PAH degradation in soils when amphiphiles are added above their critical micellar concentration [3, 4], while other authors claim an inhibition when micellar aggregates are present [5]. Differences in culture composition and a wide variety of experimental conditions probably account for the apparent inconsistency of the data reported. The toxicity of the substrates and/or the inhibition exerted by the products should be always considered.

Since PAHs are generally found in mixtures at contaminate sites, it is fundamental to investigate the solubilization of PAHs from mixtures in the presence of surfactant. The amount of individual PAH and other 
compounds when solubilized from mixtures is not related in a simple manner to their single solute solubilities. Studies have reported that in the presence of a co-solute, the amount of organic compounds in micellar solutions has been found to decrease or increase compared to single components system [6-9].

Also the rate these contaminants are transferred to the aqueous phase and the factors that control them are important to evaluate the impact on the biodegradation rates and bioavailability [10]. Some studies have reported rates of solubilization of organic compounds from solid and liquid phases [4, 11-13]. The knowledge of the ratelimiting mechanisms is thus a key to the understanding of the critical factors that influence the mechanism of NAPL solubilization by surfactant solution.

Interfacial mass transfer kinetic measurements require the simultaneous defining and monitoring of interfacial area and volume as a function of time. Few experimental methods that have been applied to the study of solubilization kinetics have been well defined in these terms. Some attempts have been made to overcome these difficulties. Some studies have reported rates of solubilization of organic compounds from solid and liquid phases [3, 12-16] and emulsions [16] using various experimental systems such as drop-on-fiber or oil droplet [14], laminar flow system [13], completely mixed batch systems [3], and solid and liquid rotating disks [12, 15].

The main objective of this work is to investigate the biodegradation of PAHs from multicomponent NAPLs in the presence of a nonionic surfactant Brij 35 using a rotating disk apparatus. A rotating disk apparatus, which the NAPL-water interfacial area was maintained constantly by a membrane, was conceived and successfully used to investigate the mass transfer of naphthalene and phenanthrene from a non-aqueous phase liquid into a nonionic surfactant solution $[17,18]$.

The majority of studies investigated the effects of surfactant on biodegradation and bioavailability of PAHs in biotic systems with inadequate assessment of concurrent dissolution and biodegradation processes, which makes it difficult to elucidate the effect of surfactants on biodegradation and explain the causes of the observed results. A complete characterization of mass transfer and equilibrium partitioning phenomena in the test systems has been done in the present study. First, equilibrium solubilization experiments were performed to improve the understanding of partitioning of PAHs in the nonionic surfactant solutions in multi-solute systems and to quantify equilibrium surfactant solubilization of target PAHs. Next, the kinetics of solubilization of PAHs in the Brij 35 solutions from NAPLs was studied [17, 18]. Finally, with prior assessment of solubilization, mass transfer process and the mechanisms that control the dissolution of PAHs in NAPL-surfactant systems, the effects of the surfactant on the biodegradation extent and rates were investigated.

Moreover, most studies on solubilization kinetics and biodegradation of PAH in surfactant system have investigated single pure compound system with few exceptions such as Thiem et al. [19] that evaluate PAH biodegradation from coal-tar in soil. However, real NAPLs are a complex mixture containing hundreds of components. Experimental investigations of complex multicomponent NAPLs are often limited by the analytical difficulties that involve complete characterization of composition and bulk properties, making it difficult to draw inferences from experimental data. In addition many of the compounds may be toxic to the microorganisms. A synthetic NAPL containing PAHs for which the complete composition is readily determined can be used as a model organic liquid that is representative of coal tar or creosote, without involving the uncertainties associated with field samples.

The results of the research will help identify appropriate surfactants for use in remediation at NAPL-contaminated sites and provide a rational basis for selecting the optimum surfactant and its dose for enhanced solubilization of PAHs from NAPLs.

\section{Materials and methods}

A detailed description of the experimental apparatus, materials and measuring techniques for the dissolution experiments is given elsewhere [17, 18, 20, 21]. Here we will present a brief summary of the experimental aspects of the work.

NAPL and surfactant solution preparation

Naphthalene (purity greater than $99 \%$, melting point equal to $80.2{ }^{\circ} \mathrm{C}$ ) phenanthrene (purity greater than $98 \%$, melting point equal to $99.2^{\circ} \mathrm{C}$ ) $n$-hexadecane (purity greater than $99.9 \%$, melting point equal to $18.1^{\circ} \mathrm{C}$ ) HPLC-grade methanol, and non-ionic surfactant Brij 35 were purchased from Sigma Aldrich Chemical Co. and were used without further purification. The solubility limit for hexadecane was less than $0.9 \times 10^{-6}$ and equal to 31 and $1.12 \mathrm{mg} / \mathrm{L}$ for naphthalene and phenanthrene, respectively. Some relevant properties of the chemicals used in this study are presented in Table 1. Brij 35 is the commercial name for $\mathrm{CH}_{3}\left(\mathrm{CH}_{2}\right)_{11}\left(\mathrm{OCH}_{2} \mathrm{CH}_{2}\right)_{23} \mathrm{OH}$, a dodecyl-poly-ethyleneoxide-ether. The number 35 refers to the sum of the length of the alkyl chain (12) and the ethylene-oxide groups (23). Nonionic surfactants containing poly-ethylene-oxide chains as the hydrophilic part and n-alkyl 
Table 1 Physicochemical properties of NAPL components

\begin{tabular}{lllllll}
\hline Target compound & Other solute & $\begin{array}{l}\text { Mol. Wt. } \\
(\mathrm{g} / \mathrm{mol})^{\mathrm{a}}\end{array}$ & $\begin{array}{l}\text { Solid/liquid } \\
\text { fugacity ratio }^{\mathrm{b}}\end{array}$ & Log $K_{\mathrm{ow}}$ & $\begin{array}{l}\mathrm{C}_{\mathrm{AQ} \text {, sat }}^{i} \\
(\mu \mathrm{mol} / \mathrm{L})^{\mathrm{d}}\end{array}$ & $K_{N}^{i}(\mu \mathrm{mol} / \mathrm{L})^{\mathrm{d}}$ \\
\hline Hexadecane & - & 226.45 & 1 & $6.6^{\mathrm{c}}$ & - & - \\
Naphthalene & None phenanthrene & 128.19 & 0.306 & $3.36^{\mathrm{a}}$ & $228.7 \pm 9.7$ & $1,240 \pm 43.4(1,172 \pm 36.9)^{\mathrm{e}}$ \\
Phenanthrene & None naphthalene & 178.24 & 0.279 & $4.57^{\mathrm{a}}$ & $6.32 \pm 0.22$ & $85.5 \pm 2.9(97.7 \pm 5.4)^{\mathrm{e}}$ \\
\hline
\end{tabular}

${ }^{a}$ Schwarzenbach et al. [27]

b Mukherji et al. [28]

c Chiou and Schmedding [29]

d Hill and Ghoshal [9]

e Values of $K_{N}^{i}$ in the presence of the other PAH solute in a three-component NAPL

chains as the hydrophobic part form micelles in water [22]. The average molecular weight is equal to $1,198 \mathrm{~g} /$ mol, Critical Micellar Concentration (CMC) equal to $9.2 \times 10^{-5} \mathrm{~mol} / \mathrm{L}$, hydrophilic-lipophilic balance equal to 16.9. If another solvent (e.g., an alcohol) is added to the binary Brij/water system, then microemulsions with many interesting properties are obtained. The systems have practical applications in cosmetics, pharmaceuticals, paints, cleaning, and biotechnology. Brij 35 is useful for the solubilization of membrane proteins and can also entrap hydrophobic reactants in liquid-phase enzyme reactions. Surfactant Brij 35 is one of the most commonly used non-ionic surfactant employed in many experimental studies on solubilization and biodegradation of hydrophobic organic compounds [9, 17, 18, 20, 21].

The NAPLs were synthesized by dissolving various quantities of individual crystalline PAHs with hexadecane in sealed Pyrex vials and mixing in a rotary shaker. Surfactant solutions were prepared by mixing a known weight of surfactant into $1 \mathrm{~L}$ of deionized water and stirring for $24 \mathrm{~h}$.

Equilibrium partitioning experiments

Two $\mathrm{mL}$ of NAPL was added to $25 \mathrm{~mL}$ of surfactant solution in $40 \mathrm{~mL}$ glass vials sealed with Teflon-lined caps. For each surfactant, solutions prepared at specific surfactant doses were contacted with NAPLs of varying PAH content in a series of vials. The vials were mounted at on an orbital shaker set at $150 \mathrm{rpm}$ for $96 \mathrm{~h}$. Segregated NAPL and micellar surfactant solution phases were maintained during mixing. After equilibration, aliquots of $1 \mathrm{~mL}$ were taken from aqueous phase in each vial using a syringe, centrifuged, diluted with HPLC-grade methanol and transferred to a 2-mL HPLC vial. All glassware was acidwashed and rinsed with water prior to use. All experiments were conducted in a temperature-controlled walk-in chamber at $25 \pm 1{ }^{\circ} \mathrm{C}$.
Dissolution and biodegradation experiments

The rotating apparatus consisted in a $1 \mathrm{~L}$ jacketed glass vessel heated by a thermostat bath to control the temperature at $25 \pm 0.1{ }^{\circ} \mathrm{C}$. A glass-spinning cell coupled to a glass shaft of a mechanical stirrer with constant velocity motor was glued to a microporous polypropylene membrane to maintain constant the interfacial area. Thus the membrane wetted by the non-aqueous phase liquid formed the 'rotating disk' for mass transfer.

Biodegradation experiments were carried out under aerobic conditions in the absence and presence of surfactant at doses equal to 5 and 25 times the CMC. Higher doses of surfactants were not used to avoid toxic effects to the cells. The experiments employed batch reactor containing segregated NAPL and micellar solution phases.

The relative amounts of the PAHs were varied to obtain NAPLs of different composition. Three combinations of mole fractions were used with naphthalene varying from $0.01,0.09$ to 0.17 and phenanthrene varying from 0.01 , 0.03 to 0.05 . A total of $250 \mathrm{~mL}$ of liquid solution (pure mineral solution or mineral solution with surfactant) was used.

The strain was maintained on slants with R2A agar (Difco) supplemented with phenanthrene. Mineral salts medium was made by combining $1 \mathrm{~g} \mathrm{KH}_{2} \mathrm{PO}_{4}, 0.86 \mathrm{~g}$ $\mathrm{Na}_{2} \mathrm{HPO}_{4}, 1 \mathrm{~g} \mathrm{NH}_{4} \mathrm{Cl}, 0.06 \mathrm{~g} \mathrm{MgSO}_{4}, 0.06 \mathrm{~g} \mathrm{CaCl}_{2} \cdot 2 \mathrm{H}_{2} 0$ and $1 \mathrm{~mL}$ trace metal solution of deionized water. Sterile stock trace metals solution was made of $3.3 \mathrm{mg} / \mathrm{L}$ $\mathrm{MnSO}_{4} \cdot \mathrm{H}_{2} \mathrm{O}, 6.2 \mathrm{mg} / \mathrm{L} \mathrm{CuSO}_{4} \cdot 5 \mathrm{H}_{2} \mathrm{O}, 7.60 \mathrm{mg} / \mathrm{L} \mathrm{ZnSO}_{4}$. $7 \mathrm{H}_{2} \mathrm{O}, 11.7 \mathrm{mg} / \mathrm{L} \mathrm{Na}_{2} \mathrm{MoO}_{4} \cdot 2 \mathrm{H}_{2} \mathrm{O}, 64.6 \mathrm{mg} / \mathrm{L} \mathrm{FeSO}_{4} \cdot 7 \mathrm{H}_{2} \mathrm{O}$ and $1 \mathrm{~L} \mathrm{HCl}$ of $0.05 \mathrm{~mol} / \mathrm{L} \mathrm{HCl}$.

The microorganism selected was the Pseudomonas stutzeri P-16 isolated from soils contaminated with creosote. P. stutzeri is a well-characterized bacterium chosen for its well-known ability to degrade a number of PAHs including phenanthrene and naphthalene [23-25]. The microorganism does not produce significant amount of 
extracellular emulsifying agents or biofilms [23-25]. Surface tension measurements of the bacterial growth fluid on naphthalene or phenanthrene did not involve the production of biosurfactants [23].

In $50 \mathrm{~mL}$ culture flasks, from a frozen glycerol stock of P. stutzeri P-16 into $25 \mathrm{~mL}$ of mineral medium were prepared. After inoculation, the flask was placed on an orbital shaker and agitated at $150 \mathrm{rpm}$ at $25^{\circ} \mathrm{C}$ overnight. After, the solution was diluted to a final volume of $250 \mathrm{~mL}$, supplemented with phenanthrene and growth was followed by periodically measurement to a final optimal absorbance of 0.1 . The bacterial cells growing in the culture media were harvested, washed with the buffer solution and resuspended in the same buffer. The cells in the culture tubes were thoroughly mixed by mounting the tube onto a vortex mixer and were transferred with pipettes with sterile tips into the reactor.

The reactor containing an aqueous phase and preequilibrated with $10 \mathrm{~mL}$ NAPL was inoculated with $5 \mathrm{~mL}$ of the washed cells and agitated at $100 \mathrm{rpm}$. A relatively constant NAPL composition was maintained using a large volume of $10 \mathrm{~mL}$. This allowed the mole fraction of the PAHs in the NAPL to change no more than $10 \%$ as a result of solubilization by surfactant micelles and biodegradation in the aqueous phase over the $10 \mathrm{~h}$ of the experiment. Aliquots were removed at interval of time and the growth of bacteria was measured by optical density and the concentrations of the PAHs were measure by HPLC. Aqueous phase samples were taken at different times, put into $10 \mathrm{~mL}$ sealed Pyrex centrifuge tubes, diluted with HPLC methanol grade and centrifuged at 4,000 rpm for $15 \mathrm{~min}$. Supernatant samples were used for PAHs analysis by HPLC. Aerobic conditions were maintained by purging briefly the system with oxygen at the time of sampling. All glassware was acid-washed and rinsed with water prior to use.

Growth curves of bacteria in the presence and absence of surfactant were performed. Culture flasks were filled with $30 \mathrm{~mL}$ of solution consisting of mineral medium and a certain volume of solution of Brij 35 to attain the specific doses of 5 and 25 times the CMC. Measuring the optical density at $420 \mathrm{~nm}$ followed growth of bacteria.

\section{Analytical methods}

Naphthalene and phenanthrene were analyzed using an Agilent 1100 series HPLC, equipped with a diode array (DAD) detector set at wavelength equal to $240 \mathrm{~nm}$ and fluorescence (FLD) detector set at excitation $280 \mathrm{~nm}$ and emission $389 \mathrm{~nm}$ wavelength. A $250 \mathrm{~mm}$ x $2.1 \mathrm{~mm}$ reverse phase Vydac column (Model 201TP52) was employed. The HPLC was programmed for an acetonitrile-water elution at a constant flow rate of $1 \mathrm{ml} / \mathrm{min}$ and a constant temperature of $35{ }^{\circ} \mathrm{C}$ over a 33 -min program run. Acetonitrile $(60 \%)$ and water was used as the mobile phase.

Growth of $P$. stutzeri P-16 was monitored by measuring optical density at $420 \mathrm{~nm}$ with a double-beam UV/vis scanning spectrophotometer. One absorbance unit corresponded to $500 \mathrm{mg}$ (dry weight) of biomass per liter [25, 26].

\section{Results and discussion}

Equilibrium partitioning experiments

The micelle-water equilibrium partition coefficient, $K_{M}^{i}\left(\mathrm{~L}^{3} / \mathrm{M}\right)$, for PAHs in the three-component NAPL and micellar surfactant solution systems was calculated as follows $[9,20]$ :

$K_{M}^{i}=\operatorname{MSR}^{i} /\left(K_{N}^{i} \times X_{N}^{i}\right)=\operatorname{MSR}^{i} / C_{\mathrm{AQ}}^{i}$

where $K_{N}^{i}\left(\mathrm{M} / \mathrm{L}^{3}\right)$ is the Raoult's Law based ratio of the equilibrium concentration of solute $i$ in the true aqueous phase, $C_{\mathrm{AQ}}^{i}\left(\mathrm{M} / \mathrm{L}^{3}\right)$, and the mole fraction of the solute in the NAPL, $X_{N}^{i}$, and MSR ${ }^{i}$ is the ratio of number of moles of solute $i$ per mole of surfactant in the micellar phase.

The micelle-water equilibrium partition coefficient, $K_{M}^{i}$, for PAHs in the three-component NAPL and micellar surfactant solution systems was calculated as Eq. 1. In this study Eq. 1 was fitted to a large $\operatorname{MSR}^{i}$ and $X_{N}^{i}$ dataset obtained from systems containing three-component NAPLs comprised of naphthalene, phenanthrene and hexadecane. $\mathrm{MSR}^{i}$ was determined as the slope of the linear plot of the total concentration of the PAH in the bulk aqueous phase versus the corresponding surfactant concentration above the CMC. The $K_{N}^{i}$ of each PAH is presented in Table 1.

Figure 1 presents the partition coefficients of PAHs in systems where NAPLs with various PAH mole fractions were contacted with different surfactant solutions. The extent of selective solubilization of naphthalene and phenanthrene in different surfactant solutions was determined by comparing the $K_{M}^{i}$ of each solute in the threecomponent NAPL with those obtained from single-solute, two-component NAPL systems. The $K_{M}^{\text {naph }}$ values were obtained for a series of NAPLs of $X_{N}^{\text {naph }}=0.17$ and varying $X_{N}^{\text {phen }}$, and the $K_{M}^{\text {phen }}$ values were obtained for a series of NAPLs with $X_{N}^{\text {phen }}=0.05$ and varying $X_{N}^{\text {naph }}$. The $K_{M}^{i}$ values for other NAPLs are not shown, but identical trends were noted. In the first plot the origin corresponds to the values of the partition coefficient for two-component system (just naphthalene in hexadecane for the first plot and just phenanthrene in hexadecane for the second plot) and the region in the bracts corresponds to the coefficients in the three-component NAPL. Also from a comparison of the $K_{M}^{i}$ values obtained from fitting Eq. 1 to the 


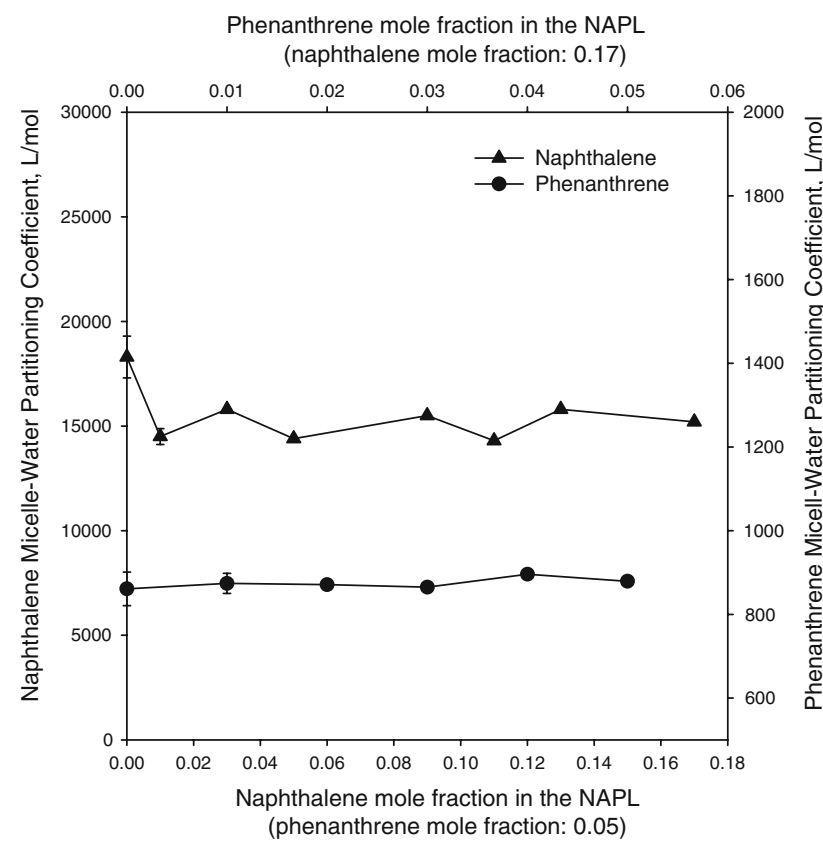

Fig. 1 NAPL-surfactant micelle partitioning coefficients $\left(K_{M}^{i}\right)$. Error values indicate $95 \%$ confidence intervals

experimental data, for each PAH of the three-component NAPL containing naphthalene and phenanthrene, with those for either PAH in the two-component NAPLs it can be observed the effects of co-solubilization. By comparing the $K_{M}^{i}$ of each solute in the NAPL with those obtained from single-solute systems the coefficients of one PAH were affected by the presence of the other. In surfactant solutions of Brij 35 the solubilization of phenanthrene decreased in the presence of naphthalene. The $K_{M}^{\text {naph }}$ values from the two- and three-component system were very similar; however, the $K_{M}^{\text {phen }}$ values are consistently lower in the three-component NAPL as compared to the two-component NAPL. Note that the scales of the PAHs are different and the variations are very significant in the phenanthrene plot.

\section{Dissolution experiments}

A detailed description of the results for the dissolution experiments is given elsewhere $[17,18]$. The dissolution of
PAHs into pure water and into solution of Brij 35 across the NAPL-wetted membrane was measured at doses varying from 1 to 100 times the CMC [18]. The total PAHs concentration in the bulk aqueous phase at various times in reactor were used to determine the experimental mass transfer coefficients, $k^{i}[\mathrm{~L} / \mathrm{T}]$ by fitting the integrated form of the first-order rate model, $\mathrm{d} C_{T}^{i} / \mathrm{d} t=k_{f}^{i} a\left(C_{T, \text { eq }}^{i}-C_{T}^{i}\right)$ to experimental data [14]. In this equation, $C_{T, \mathrm{eq}}^{i}$ is the equilibrium concentration of solute $i$ in the bulk solution, $C_{T}^{i}$ is the contaminant concentration in the bulk solution. The total equilibrium saturation concentration of solute is a function of its equilibrium concentration in the aqueous phase alone, $C_{a, \text { eq }}^{i}$ and surfactant concentration, $C_{\text {surf }}$ depends on both the $\mathrm{CMC}$ and $\mathrm{SC}$, the solubilization capacity $[18,21]$. Note that the input values for the above equation of equilibrium concentration for naphthalene and phenanthrene in Brij 35 solutions obtained from the partitioning experiments $[17,18]$. An important decrease in the mass transfer coefficients followed by an approximately constant coefficient was observed as shown in Table 2; however, the rate of solubilization of the PAHs increased due to the increasing driving force term that becomes much larger at higher surfactant concentration. As a result, a higher surfactant concentration increases not only the total amount of PAHs in the aqueous bulk phase, but also the maximum rates of solubilization. The same trend has been also reported in the literature $[3,17,18]$.

Experiments were also performed for solutions of Brij 35 to identify the potential rate-limiting mechanisms at three rotation speeds equal to 100,120 and $150 \mathrm{rpm}$ for each dose of surfactant [17]. As demonstrated by Bernardez and Ghoshal [17] the results adjusted to the prediction of the model developed by Chan et al. [12] and demonstrated that the solubilization rate was controlled by the rate-limiting mechanisms of micelle desorption and diffusion of loaded micelles.

Experiments in pure water at different rotations demonstrated that the rotating apparatus built for the experiments behaved as a Levich's rotating solid disk where the liquid is entrained by a rotating disk whose axis is perpendicular to its plane surface and defined an equation as follows $[14,15]$ :

Table 2 Mass transfer coefficient for naphthalene and phenanthrene dissolution from a multicomponent NAPL with naphthalene mole fraction equal to 0.09 and phenanthrene mole fraction of 0.03 , as a function of surfactant concentration in aqueous solutions of surfactant Brij 35

\begin{tabular}{lll}
\hline Concentration of Brij 35 (mol/L) & $k_{\text {naphthalene }}(\mathrm{m} / \mathrm{s})$ & $k_{\text {phenanthrene }}(\mathrm{m} / \mathrm{s})$ \\
\hline $9.2 \times 10^{-5}$ & $1.22 \times 10^{-5} \pm 3.5 \times 10^{-7}$ & $8.82 \times 10^{-6} \pm 3.0 \times 10^{-7}$ \\
$2.3 \times 10^{-3}$ & $1.10 \times 10^{-5}$ & $5.73 \times 10^{-6}$ \\
$4.6 \times 10^{-3}$ & $7.94 \times 10^{-6}$ & $4.12 \times 10^{-6}$ \\
$9.2 \times 10^{-3}$ & $7.14 \times 10^{-6} \pm 2.2 \times 10^{-7}$ & $4.10 \times 10^{-6} \pm 8.0 \times 10^{-8}$
\end{tabular}

Error bars represent the standard deviation 
$S h=0.62(R e)^{1 / 2}(S c)^{1 / 3}$

The Reynolds $(R e)$ and Schmidt $(S c)$ numbers are defined as $R e=R^{2} \omega \rho / \mu$ and $S c=\mu / \rho D$, respectively were $\rho$ is the density $\left(\mathrm{ML}^{-3}\right)$ and $\mu$ is the viscosity $\left(\mathrm{ML}^{-1} \mathrm{t}^{-1}\right)$. The $D$ is the diffusion coefficient of solute $i\left(L^{2} t^{-1}\right) ; k_{f}^{i}$ is the mass transfer coefficient for solute $i$ dissolution into aqueous phase $\left(L t^{-1}\right) ; R$ is the disc's radius $(L) ; v$ is the kinetic viscosity $\left(L^{2} t^{-1}\right)$; $\omega$ is the disc's rotational speed $\left(\operatorname{radt}^{-1}\right)$; and $S h$ is the Sherwood number.

Comparing the theoretical predictions by the Levich's equation with the experimental in general, a good agreement was obtained, suggesting that the liquid disk behaves similarly to a rotating solid disk (Table 3 ). The deviations are attributed to the imperfections of the apparatus and especially the roughness of the surface of the disk due to the membrane compared to the delicate precision stainless steel apparatus without imperfections at the surface. The experimental mass transfer coefficients, $k^{i}[\mathrm{~L} / \mathrm{T}]$ were of naphthalene and phenanthrene in the water and were respectively equal to $5.55 \times 10^{-3}$ and $2.96 \times 10^{-3} \mathrm{~cm} / \mathrm{s}$.

\section{Biodegradation experiments}

The effect of surfactant dose on the biodegradation of PAHs from the NAPL was initially investigated for solutions at dose equal to 5 and 25 times the CMC. The absence of toxicity due the addition of surfactants was observed. The growth curves of $P$. stutzeri P-16 were not affect by the presence of surfactants used (Fig. 2).

A NAPL with naphthalene mole fraction of 0.09 $(0.45 \mathrm{~g})$ and phenanthrene mole fraction of $0.03(0.21 \mathrm{~g})$ in $10 \mathrm{~mL}$ of hexadecane was placed in the spinning cell of the rotating reactor. After both PAHs equilibrated with the pure mineral medium the cells were inoculated and the $\mathrm{PAH}$ concentrations in the aqueous phase were monitored over time. The concentration of naphthalene decreased to $60 \%$ of the initial value in less than $3 \mathrm{~h}$ and the phenanthrene concentration decreased rapidly over time and reached a plateau at $0.08 \mathrm{mg} / \mathrm{L}$ resulting in a decreasing of $80 \%$ of the initial value (Fig. 3). The dissolution and

Table 3 Comparison of theoretical and experimental values for the rotating apparatus for naphthalene and phenanthrene in pure water

\begin{tabular}{llllll}
\hline$R e^{0.5}$ & \multicolumn{2}{l}{ Naphthalene } & & \multicolumn{2}{l}{ Phenanthrene } \\
\cline { 2 - 3 } & $S h$ & $S h_{\exp }$ & & $S h$ & $S h_{\exp }$ \\
\hline 35.45 & 242.0 & 284.0 & & 289.6 & 265.5 \\
45.76 & 312.4 & 363.0 & & 373.8 & 359.0 \\
57.90 & 395.2 & 435.0 & & 473.0 & 530.0 \\
66.32 & 452.7 & 470.0 & & 541.8 & 577.0 \\
70.91 & 484.0 & 449.0 & & 579.3 & 455.0 \\
\hline
\end{tabular}

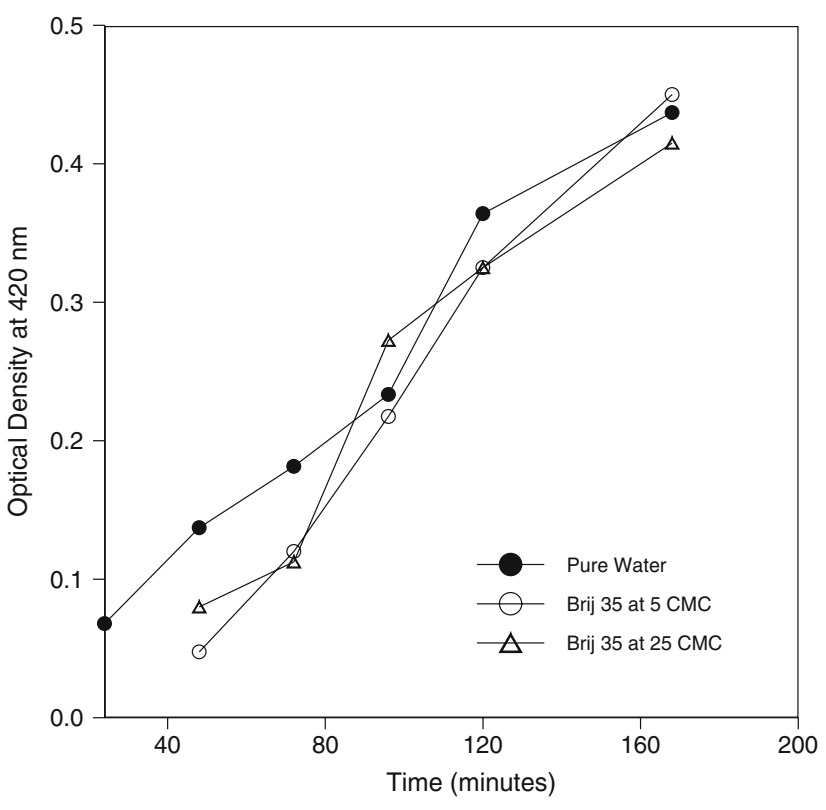

Fig. 2 Growth kinetics of Pseudomonas stutzeri P-16 on phenanthrene in pure mineral medium in absence of surfactant (filled circles), or in the presence of Brij 35 at $5 \mathrm{CMC}$ (diamonds) and 25 CMC (triangles). Experiments were conducted at $25^{\circ} \mathrm{C}$ in conical flasks with agitation at $150 \mathrm{rpm}$

subsequent biodegradation of naphthalene and phenanthrene in solutions of Brij 35 at concentration equal to 5 CMC was investigated (Fig. 3). During the time period PAH concentrations in the bulk aqueous phase decreased, the growth curve of $P$. stutzeri P-16 varied from exponential at the very beginning to linear. Fitting an exponential curve to the experimental data, for the first $60 \mathrm{~min}$, identified the exponential characteristics.

The initial rates of biodegradation for both PAHs were then calculated from the slope of the first $30 \mathrm{~min}$ of the plots from the total PAHs concentration as a function of the time (Table 4). In pure mineral medium the rates of biodegradation for naphthalene and phenanthrene were respectively equal to 0.034 and $0.0074 \mathrm{mg} / \mathrm{L}$ min. Brij 35 when present at doses equal to $5 \mathrm{CMC}$ significantly increased the initial rates of biodegradation of PAHs as compared to the rates in pure water. The naphthalene and phenanthrene biodegradation rates were three and almost seven times higher when compared to pure mineral medium with naphthalene being equal to 0.1 and phenanthrene equal to $0.05 \mathrm{mg} / \mathrm{L} \mathrm{min}$.

A rapid decrease in bulk aqueous phase concentration at initial stages of biodegradation experiments was observed for both PAHs. In the initial period, the system is preequilibrated and the PAH concentrations in the bulk aqueous phase are higher and thus can be easily degraded by the bacteria. However, at a later stage, when the concentrations of the PAH were low, a plateau was observed. 


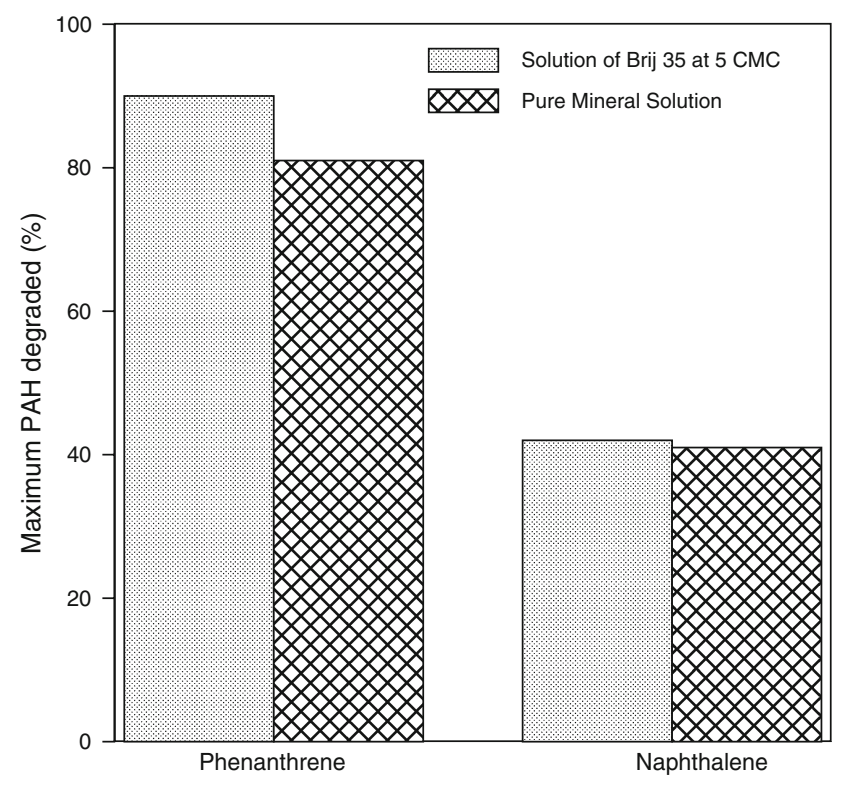

Fig. 3 Naphthalene and phenanthrene dissolution and biodegradation from NAPL of composition corresponding to naphthalene mole fraction equal to 0.09 and phenanthrene of 0.03 in mineral medium containing no surfactant and in solution of surfactant Brij 35 at 5 $\mathrm{CMC}$

Table 4 Initial biodegradation rates for PAHs in a surfactant free system and in a surfactant solution system at a dose equal to $5 \mathrm{CMC}$ containing a NAPL with naphthalene mole fraction equal to 0.09 and phenanthrene mole fraction equal to 0.03

\begin{tabular}{lll}
\hline System investigated & \multicolumn{2}{l}{ Initial biodegradation rates $(\mathrm{mg} / \mathrm{L}$ min $)$} \\
\cline { 2 - 3 } & Phenanthrene & Naphthalene \\
\hline Water & 0.0074 & 0.034 \\
Brij 35 & 0.050 & 0.10 \\
\hline
\end{tabular}

At this latter stage, dissolution of PAHs limits the formation of biomass and linear growth of P. stutzeri P-16 is observed. Active biodegradation occurs during this stage because for the PAH concentration to be constant its biodegradation rate has to match solubilization rate.

In the scenarios investigated where the amount of the polycyclic aromatic hydrocarbons partitioned into the micelles increased with surfactant dose the extent and rates of biodegradation were inhibited. The same was observed with NAPL composition.

For example, in solutions of Brij 35 at low surfactant dose equal to $5 \mathrm{CMC}$ and at low mole fraction of PAHs equal to 0.01 , naphthalene and phenanthrene in the bulk phase were completely degraded within 30 and $240 \mathrm{~min}$, respectively (Fig. 4). At the highest dose of surfactant equal to $25 \mathrm{CMC}$, at same NAPL composition, only $54 \%$ of naphthalene and $70 \%$ of the initial phenanthrene concentration were degraded after $480 \mathrm{~min}$. Experiments were performed at $5 \mathrm{CMC}$ but with naphthalene and

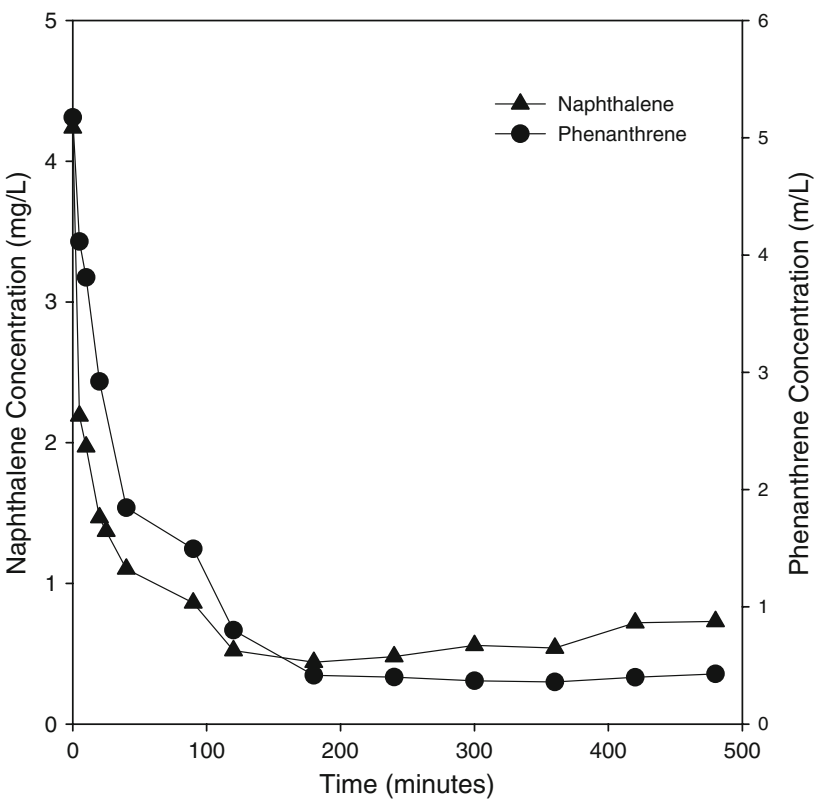

Fig. 4 Naphthalene (triangles) and phenanthrene (circles) dissolution and biodegradation from NAPL of composition corresponding to naphthalene and phenanthrene mole fraction equal to 0.01 in solution of surfactant Brij 35 at $25 \mathrm{CMC}$. Lines connecting points have been drawn to aid the visualization

phenanthrene at the highest mole fraction equal to 0.17 and 0.05 (data not shown). When the mole fraction of the PAHs in the NAPL increased, the extent of degradation significantly decreased. The same trend was observed when the biodegradation in solutions at $25 \mathrm{CMC}$ at lowest NAPL composition is compared to the biodegradation in system containing a NAPL at highest mole fraction.

The rates of biodegradation were lower in solutions of Brij 35 at $25 \mathrm{CMC}$ than in solutions at $5 \mathrm{CMC}$ despite the increase in the rates of solubilization (Table 5). The increase in surfactant dose enhanced the PAH solubilization rate in the bulk aqueous phase by increasing the NAPL solubilization rate plus PAH incorporation into micelles. The rates of solubilization of Brij 35 at 25 CMC were almost four times higher than the rates in the solution at 5 CMC (Table 5). However, the biodegradation rates were not similarly enhanced but decreased. The results at different surfactant concentrations show that besides the stimulation of the biodegradation rates an increase of surfactant dose may have a negative impact on the rates of biodegradation.

A decrease in the rates of biodegradation with the increase of the amount of the PAHs in the non-aqueous liquid phase may be related to the toxicity of these compounds to the microorganisms.

To provide information about the mechanism of up-take by the bacteria comparisons of the experimental biodegradation rates with those predicted by a model based on the 
Table 5 Initial solubilization and biodegradation rates for phenanthrene in Brij 35 solution system containing NAPL with naphthalene and phenanthrene at different mole fractions

\begin{tabular}{llllll}
\hline CMC & \multicolumn{2}{l}{ Target PAH: phenanthrene } & & \\
\cline { 2 - 3 } & \multicolumn{2}{l}{ Solubilization rate $(\mathrm{mg} / \mathrm{L} \mathrm{s})$} & & \multicolumn{2}{l}{ Biodegradation rate $(\mathrm{mg} / \mathrm{L} \mathrm{s})$} \\
\cline { 2 - 3 } \cline { 5 - 6 } & $X_{\mathrm{NAPL}}^{\text {phen }} 0.01$ & $X_{\mathrm{NAPL}}^{\text {phen }} 0.05$ & & $X_{\mathrm{NAPL}}^{\text {phen }} 0.01$ & $X_{\mathrm{NAPL}}^{\text {phen }} 0.05$ \\
\hline 5 & $1.2 \times 10^{-5}$ & $8.74 \times 10^{-6}$ & & $1.68 \times 10^{-3}$ & - \\
25 & $5.2 \times 10^{-5}$ & $1.20 \times 10^{-5}$ & & $1.36 \times 10^{-3}$ & $2.79 \times 10^{-5}$ \\
\hline
\end{tabular}

assumption that solutes must be first dissolved in the aqueous phase to be available to bacteria were performed. The model coupled two differential equations for the input rate of PAHs into the bulk aqueous phase due to the dissolution and for the rate of removal from the aqueous phases due to degradation.

$V \frac{\mathrm{d} C_{T}^{i}}{\mathrm{~d} t}=k_{f}^{i} a\left(C_{T, \mathrm{eq}}^{i}-C_{T}^{i}\right)-\left.V \frac{\mathrm{d} C_{T}^{i}}{\mathrm{~d} t}\right|_{\mathrm{bio}}$

$\frac{\mathrm{d} X}{\mathrm{~d} t}=-\left.Y_{i} \frac{\mathrm{d} C_{T}^{i}}{\mathrm{~d} t}\right|_{\text {bio }}$

Some considerations were taken such as: the $P$. stutzeri P-16 degrade PAHs according to Michaelis-Menton or Monod kinetics [3, 24, 25] and the aqueous PAHs concentration, $C_{a}^{i}$, can be related to the total liquid phase concentration, $C_{T}^{i}$ as described by Grimberg et al. [3] with $C_{a}^{i}=C_{T}^{i}\left(C_{a, \mathrm{eq}}^{i} / C_{T, \mathrm{eq}}^{i}\right)$. In the above equations $Y$ stands for yield coefficient, $\left(\mathrm{MM}^{-1}\right), V$ stands for volume of aqueous phase, $\left(\mathrm{L}^{3}\right)$ and $X$ stands for biomass concentration, $\left(\mathrm{ML}^{-3}\right)$.

The overall mass transfer coefficient is the addition of the two individual processes including the mechanisms of desorption and diffusion of the micelles containing the PAHs determined from previous dissolution experiments [17]. The total saturation concentration, $C_{T \text {, eq }}^{i}$, is invariant over the time of the experiments. A fourth-order RungeKutta algorithm is used to solve the system where the initial conditions are $C_{T(t=0)}^{i}=C_{T, o}^{i}$ and $X_{(t=0)}=X_{o}$.

The required model parameters are quantified from the independent equilibrium, mass transfer and biodegradation experiments obtained from the batch experiments, while the yield coefficients and Monod's parameters are obtained from published parameters of phenanthrene and naphthalene biodegradation experiments by $P$. stutzeri $\mathrm{P}-16$ [3, 24, 25]. The micelle-water partitioning coefficients are present in Fig. 1 and the mass transfer coefficients are presented in Table 2. The input parameters for $P$. stutzeri $\mathrm{P}-16$ growth coefficients are presented in Table 6.

Because the results of the present study suggest that in the presence of both PAHs naphthalene degradation by $P$. stutzeri $\mathrm{P}-16$ at latter stages was not effective as
Table 6 Input parameters for $P$. stutzeri P-16 growth simulations

\begin{tabular}{ll}
\hline Biokinetic parameters $^{\mathrm{a}}$ & Value \\
\hline$q_{\max }(\mathrm{L} / \mathrm{min})$ & 0.003 \\
$K_{\mathrm{S}}(\mathrm{mg} / \mathrm{L})$ & 0.24 \\
$Y(\mathrm{~g} / \mathrm{g})$ & 1.30 \\
\hline
\end{tabular}

a Stringfellow and Aitken [23]

phenanthrene, only phenanthrene experimental data will be compared to the curve predicted by the dissolution-degradation model. The results of the comparisons of the experimental data of phenanthrene removal in the presence of surfactant Brij 35 with the model predictions are shown in Fig. 5. In general a good fitting of the model was observed and it was concluded that the contaminants appear not to be directly available to bacteria.

At surfactant dose equal to 25 times the $\mathrm{CMC}$ at lowest mole fraction in the NAPL despite the amount of naphthalene and phenanthrene degraded (90 and 95\%, respectively); the experimental data compared well with model predictions only for the initial phase over the $2 \mathrm{~h}$ of the experiment.

When PAHs are transferring from NAPL phase to the bulk aqueous phase containing surfactant, $C_{\mathrm{TOT}}^{\mathrm{i}}$, some portion partition to the true aqueous phase, $C_{\mathrm{AQ}}^{i}$ and inside the micelles, $C_{M}^{i}$. In the initial period of the biodegradation

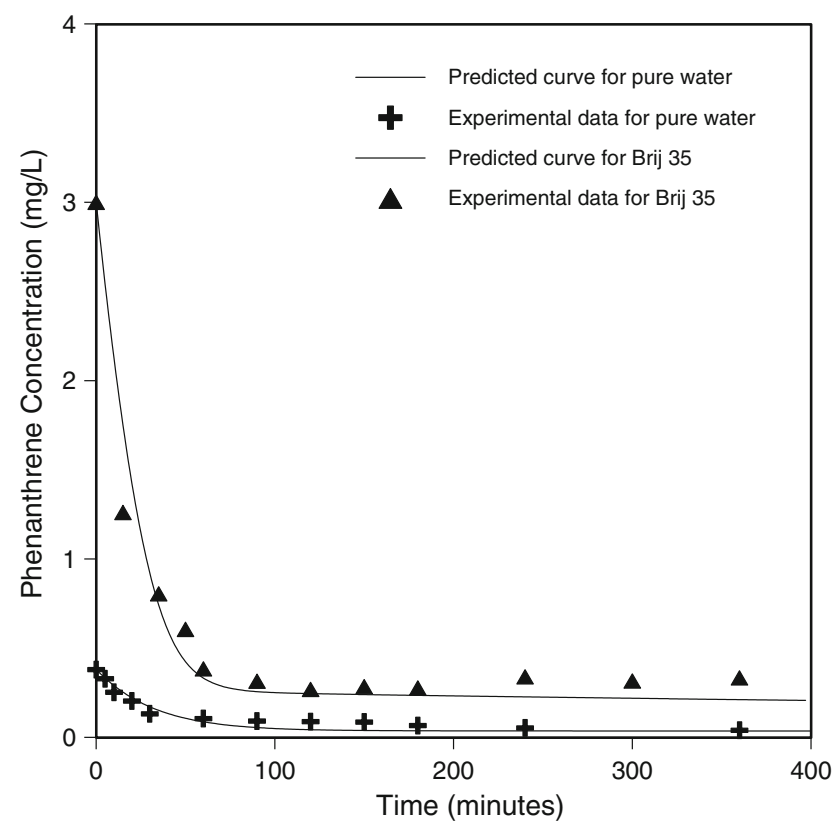

Fig. 5 Bulk phase phenanthrene concentration during batch growth of Pseudomonas stutzeri P-16 in absence of surfactant (crosses) and in the presence of Brij 35 surfactant solution at 5 times the CMC (triangles). NAPL composition corresponding to naphthalene mole fraction equal to 0.09 and phenanthrene mole fraction of 0.03 . Solid line represents the model prediction using system-specific initial conditions 
experiments the system is pre-equilibrated and the amount partitioned in the bulk aqueous phase is high. In this scenario when the microorganisms are inoculated they may easily degrade the PAHs as soon as they are dissolved in the true aqueous phase resulting in an overall enhancement of biodegradation rates. As soon as PAHs are degraded the system has to achieved equilibrium again, and if the transfer is not slowed by toxicity and/or rate-limiting mechanisms such as the solubility of the PAH compounds and the desorption and diffusion of loaded micelles the rapid replenishment of PAHs to the aqueous phase through micellar solubilization continuous.

The results suggested that the addition of surfactant resulted in an overall enhancement of biodegradation rates compared to that observed in pure aqueous solution. This is because surfactant partition significant amounts of PAHs into the bulk phase, where uptake occurs however the experiments with increasing surfactant doses suggested that the supply of PAHs to the aqueous phase through micellar solubilization may limit biodegradation rates due to the presence of increasing amounts of PAHs in the micelles compared to the amounts present in the true aqueous phase. Moreover from the dissolution experiments the rate-limiting mechanisms identified play a fundamental role during the kinetics of biodegradation. The results suggested that the mechanisms of desorption and diffusion of loaded micelles were important at high dosed of surfactants. Improve predictions of achievable rates of surfactant-aided bioremediation of PAHs in residual NAPLs at contaminated sites through development of a solubilizationdegradation model was possible.

\section{Conclusion}

A rotating disk apparatus was successfully used to investigate the biodegradation of naphthalene and phenanthrene from a non-aqueous phase liquid into a nonionic surfactant solution Brij 35. To best of our knowledge there are no reports in the literature of PAHs biodegradation experiments in NAPL-surfactant systems where a systematically and complete characterization of mass transfer and equilibrium partitioning phenomena in the test systems has been done. As a result of experimental work presented in this study a demonstration of fundamental relationship between biodegradation rate and NAPL mole fraction of $\mathrm{PAH}$, surfactant dose and the mechanisms controlling the mass transfer of PAHs from the NAPL was possible. The addition of surfactant resulted in an overall enhancement of biodegradation rates compared to that observed in pure aqueous solution because surfactant partition significant amounts of PAHs into the bulk phase, where uptake may occur but the supply of PAHs to the aqueous phase through micellar solubilization may limit biodegradation rates. It was demonstrated the relationship between biodegradation rate and the increase in the NAPL mole fraction of PAH and surfactant dose causing inhibition of the rates of biodegradation of the PAH from the NAPLs.

Acknowledgments Funding for this research was provided by Natural Sciences and Engineering Research Council (NSERC) of Canada, National Council of Science and Technology (CNPq, Brazil) and Fonds Québécois de la Recherche sur la Nature et les Technologies (FQNRT). The author also thanks Professor Stephan Grimberg of Clarkson University for providing the strain and Professor Subhasis Ghoshal of McGill University for his valuable comments.

\section{References}

1. Peters CA, Luthy RG (1993) Coal-tar dissolution in water-miscible solvents: experimental evaluation. Environ Sci Technol 27:2831-2843

2. Cerniglia CE (1990) The use of a Mycobacterium $s p$ in the remediation of polycyclic aromatic hydrocarbon wastes, Draft report: Biological remediation of contaminated sediments, with special emphasis on the great lakes, Manitowoc, Wisc., 17-19 July, pp 90-106

3. Grimberg SJ, Stringfellow WT, Aitken MD (1996) Quantifying the biodegradation of phenanthrene by Pseudomonas stutzeri $\mathrm{P} 16$ in the presence of a nonionic surfactant. Appl Environ Microbiol 62:2387-2392

4. Volkering F, Breure AM, van Andel JG, Rulkens WH (1995) Influence of nonionic surfactants on bioavailability and biodegradation of polycyclic aromatic hydrocarbon. Appl Environ Microbiol 61:1699-1705

5. Laha S, Luthy RG (1992) Effects of non-ionic surfactants on the solubilization and mineralization of phenanthrene in soil-water systems. Biotechnol Bioeng 40:1367-1380

6. Chaiko MA, Nagarajan R, Ruckenstein E (1984) Solubilization of single-component and binary mixtures of hydrocarbons in aqueous micellar solutions. J Colloid Interface Sci 99:168-182

7. Nagarajan R, Chaiko MA, Ruckenstein E (1998) Locus of solubilization of benzene in surfactant micelle. J Phys Chem 88:2916-2922

8. Guha S, Jaffé PR, Peters CA (1998) Solubilization of PAH mixtures by a nonionic surfactant. Environ Sci Technol 32:930 935

9. Hill A, Ghoshal S (2002) Micellar solubilization of naphthalene and phenanthrene from non aqueous phase liquids. Environ Sci Technol 36:3901-3907

10. Bosma NP, Middeldorp PJ, Zehnder JB (1997) Mass transfer limitation of biotransformation: quantifying bioavailability. Environ Sci Technol 31:248

11. Carroll BJ (1981) The kinetics of solubilization of nonpolar oils by nonionic surfactant solutions. Colloid Interface Sci 79:126-135

12. Chan AF, Evans DF, Cussler EL (1976) Explaining solubilization kinetics. AIChE J 22:1006-1012

13. Grimberg SJ, Nagel J, Aitken MD (1995) Kinetics of phenanthrene dissolution into water in the presence of nonionic surfactants. Environ Sci Technol 29:1480-1487

14. Cussler EL (1997) Diffusion, mass transfer in fluid systems, 2nd edn. 580 pp, Cambridge University Press, NY

15. Huang C, Evans DF, Cussler EL (1981) Linoleic acid solubilization with a spinning liquid disc. J Colloid Interface Sci 82:499

16. Weiss J, Coupland JN, Brathwaite D, McClements DJ (1997) Influence of molecular structure of hydrocarbon emulsion 
droplets on their solubilization in nonionic surfactant micelles. Colloids Surf A Physico Eng Asp 121:53

17. Bernardez LA, Ghoshal S (2008) Solubilization kinetics for polycyclic aromatic hydrocarbons transferring from a non-aqueous phase liquid to non-ionic surfactant solutions. J Colloid Interface Sci 320:298-306

18. Bernardez LA (2008) Dissolution of polycyclic aromatic hydrocarbons from a non-aqueous phase liquid into a surfactant solution using a rotating disk apparatus. Colloids Surf A Physicochem Eng Asp 320:175-182

19. Thiem A, Stieber M, Werner P, Frimmel FH (1997) Surfactantenhanced mobilization and biodegradation of polycyclic aromatic hydrocarbons in manufactured gas plant soil. Environ Sci Technol 31:2570-2576

20. Bernardez LA, Ghoshal S (2004) Selective solubilization of PAHs from a multicomponent NAPL into nonionic surfactant micelles. Environ Sci Technol 38:5878-5887

21. Bernardez LA (2008) Investigation on the locus of solubilization of polycyclic aromatic hydrocarbons in non-ionic surfactant micelles with $1 \mathrm{H}$ NMR spectroscopy. Colloids and Surf A Physicochem Eng Asp 324:71-78

22. Rosen MJ (1989) Surfactants and interfacial phenomena, 2nd edn. $431 \mathrm{pp}$, Wiley, London
23. Aitken MD, Stringfellow WT, Nagel RD, Kazunga C, Chen SH (1998) Characteristics of phenanthrene-degrading bacteria isolates from soils contaminated with polycyclic aromatic hydrocarbons. Can J Microbiol 44:743-752

24. Stringfellow WT, Aitken MD (1994) Comparative physiology of phenanthrene degradation by two dissimilar Pseudomonas isolated from a creosote-contaminated soil. Can J Microbiol 40:432438

25. Stringfellow WT, Aitken MD (1995) Competitive metabolism of naphthalene, methylnaphthalenes, and fluorine by phenanthrenedegrading Pseudomonas. Appl Environ Microbiol 61:357-362

26. Stringfellow WT, Alvarez-Cohen L (1999) Evaluating the relationship between the sorption of PAHs to bacterial biomass and biodegradation. Water Res 33:2535-2544

27. Schwarzenbach RP, Gschwend PM, Imboden DM (1993) Environmental organic chemistry. Wiley, New York

28. Mukherji S, Peters CA, Weber WJ Jr (1997) Mass transfer of polynuclear aromatic hydrocarbons from complex DNAPL mixtures. Environ Sci Technol 31:416-423

29. Chiou CT, Schmedding DW (1982) Partitioning of organic compounds in octanol-water system. Environ Sci Technol 16: $4-10$ 